\title{
MAXIMUM NORM ANALYSIS OF AN OVERLAPPING NONMATCHING GRIDS METHOD FOR THE OBSTACLE PROBLEM
}

\author{
M. BOULBRACHENE AND S. SAADI
}

Received 11 July 2005; Revised 24 September 2005; Accepted 26 September 2005

We provide a maximum norm analysis of an overlapping Schwarz method on nonmatching grids for second-order elliptic obstacle problem. We consider a domain which is the union of two overlapping subdomains where each subdomain has its own independently generated grid. The grid points on the subdomain boundaries need not match the grid points from the other subdomain. Under a discrete maximum principle, we show that the discretization on each subdomain converges quasi-optimally in the $L^{\infty}$ norm.

Copyright (c) 2006 M. Boulbrachene and S. Saadi. This is an open access article distributed under the Creative Commons Attribution License, which permits unrestricted use, distribution, and reproduction in any medium, provided the original work is properly cited.

\section{Introduction}

The Schwarz alternating method can be used to solve elliptic boundary value problems on domains which consists of two or more overlapping subdomains. The solution is approximated by an infinite sequence of functions which results from solving a sequence of elliptic boundary value problems in each of the subdomain.

Extensive analysis of Schwarz alternating method for continuous obstacle problem can be found in $[8,9]$. For convergence of discrete Schwarz algorithms of either additive or multiplicative types, see for example, $[1,6,7,11]$.

In this paper, we are interested in the error analysis in the maximum norm for the obstacle problem in the context of overlapping nonmatching grids: we consider a domain $\Omega$ which is the union of two overlapping subdomains where each subdomain has its own triangulation. This kind of discretizations is very interesting as they can be applied to solving many practical problems which cannot be handled by global discretizations. They are earning particular attention of computational experts and engineers as they allow the choice of different mesh sizes and different orders of approximate polynomials in different subdomains according to the different properties of the solution and different requirements of the practical problems. 
To prove the main result, we develop an approach which combines a geometrical convergence result due to Lions [9] and a lemma which consists of estimating the error in the $L^{\infty}$ norm between the continuous and discrete Schwarz iterates. The convergence order is then derived making use of standard finite element $L^{\infty}$-error estimate for elliptic variational inequalities.

Quite a few works on maximum error analysis of overlapping nonmatching grid methods are known in the literature (cf., e.g., $[2,3,10])$. However, to the best of our knowledge, this is the first paper that provides an $L^{\infty}$-error analysis for overlapping nonmatching grids for variational inequalities.

Now we give an outline of the paper. In Section 2. we state the continuous alternating Schwarz sequences for the obstacle problem, and define their respective finite element counterparts in the context of nonmatching overlapping grids. Section 3. is devoted to the $L^{\infty}$-error analysis of the method.

\section{The Schwarz method for the obstacle problem}

We begin by laying down some definitions and classical results related to elliptic variational inequalities.

2.1. Elliptic obstacle problem. Let $\Omega$ be a convex domain in $\mathbb{R}^{2}$ with sufficiently smooth boundary $\partial \Omega$. We consider the bilinear form

$$
a(u, v)=\int_{\Omega}(\nabla u \cdot \nabla v) d x
$$

the linear form

$$
(f, v)=\int_{\Omega} f(x) \cdot v(x) d x
$$

the right-hand side

$$
f \in L^{\infty}(\Omega)
$$

the obstacle

$$
\psi \in W^{2, \infty}(\Omega) \quad \text { such that } \psi \geq 0 \text { on } \partial \Omega \text {, }
$$

and the nonempty convex set

$$
K_{g}=\left\{v \in H^{1}(\Omega): v=g \text { on } \partial \Omega, v \leq \psi \text { on } \Omega\right\},
$$

where $g$ is a regular function defined on $\partial \Omega$.

We consider the obstacle problem: find $u \in K_{g}$ such that

$$
a(u, v-u) \geq(f, v-u), \quad \forall v \in K_{g} .
$$


Let $V_{h}$ be the space of finite elements consisting of continuous piecewise linear functions. The discrete counterpart of (2.6) consists of finding $u_{h} \in K_{g h}$ such that

$$
a\left(u_{h}, v-u_{h}\right) \geq\left(f, v-u_{h}\right) \quad \forall v \in K_{g h},
$$

where

$$
K_{g h}=\left\{v \in V_{h}: v=\pi_{h} g \text { on } \partial \Omega, v \leq r_{h} \psi \text { on } \Omega\right\}
$$

$\pi_{h}$ is an interpolation operator on $\partial \Omega$, and $r_{h}$ is the usual finite element restriction operator on $\Omega$.

The lemma below establishes a monotonicity property of the solution of (2.6) with respect to the obstacle and the boundary condition.

Lemma 2.1. Let $(\psi, g) ;(\tilde{\psi}, \tilde{g})$ be a pair of data, and $u=\sigma(\psi, g) ; \tilde{u}=\sigma(\tilde{\psi}, \tilde{g})$ the corresponding solutions to (2.6). If $\psi \geq \widetilde{\psi}$ and $g \geq \tilde{g}$, then $\sigma(\psi, g) \geq \sigma(\widetilde{\psi}, \tilde{g})$.

Proof. Let $v=\min (0, u-\tilde{u})$. In the region where $v$ is negative $(v<0)$, we have

$$
u<\tilde{u} \leq \tilde{\psi} \leq \psi
$$

which means that the obstacle is not active for $u$. So, for that $v$, we have

$$
\begin{gathered}
a(u, v)=(f, v), \\
\tilde{u}+v \leq \tilde{\psi}
\end{gathered}
$$

so

$$
a(\tilde{u}, v) \geq(f, v)
$$

Subtracting (2.10) and (2.12) from each other, we obtain

$$
a(\tilde{u}-u, v) \geq 0
$$

But,

$$
a(v, v)=a(u-\tilde{u}, v)=-a(\tilde{u}-u, v) \leq 0
$$

so

$$
v=0
$$

and consequently,

$$
u \geq \tilde{u}
$$

which completes the proof.

The proof for the discrete case is similar. 
4 Obstacle problem

Proposition 2.2. Under the notations and conditions of the preceding lemma, we have

$$
\|u-\tilde{u}\|_{L^{\infty}(\Omega)} \leq\|\psi-\tilde{\psi}\|_{L^{\infty}(\Omega)}+\|g-\tilde{g}\|_{L^{\infty}(\partial \Omega)} .
$$

Proof. Setting

$$
\Phi=\|\psi-\tilde{\psi}\|_{L^{\infty}(\Omega)}+\|g-\tilde{g}\|_{L^{\infty}(\partial \Omega)}
$$

we have

$$
\begin{aligned}
\psi & \leq \tilde{\psi}+\psi-\tilde{\psi} \leq \tilde{\psi}+|\psi-\tilde{\psi}| \leq \tilde{\psi}+\|\psi-\tilde{\psi}\|_{L^{\infty}(\Omega)} \\
& \leq \tilde{\psi}+\|\psi-\tilde{\psi}\|_{L^{\infty}(\Omega)}+\|g-\tilde{g}\|_{L^{\infty}(\partial \Omega)}
\end{aligned}
$$

hence

$$
\psi \leq \widetilde{\psi}+\Phi
$$

On the other hand, we have

$$
\begin{aligned}
g & \leq \tilde{g}+g-\tilde{g} \leq \tilde{g}+|g-\tilde{g}| \leq \tilde{g}+\|g-\tilde{g}\|_{L^{\infty}(\partial \Omega)} \\
& \leq \tilde{g}+\|g-\tilde{g}\|_{L^{\infty}(\partial \Omega)}+\|\psi-\tilde{\psi}\|_{L^{\infty}(\Omega)}
\end{aligned}
$$

so

$$
g \leq \tilde{g}+\Phi
$$

Now, making use of Lemma 2.1, we obtain

$$
\sigma(\psi, g) \leq \sigma(\widetilde{\psi}+\Phi, \tilde{g}+\Phi)=\sigma(\widetilde{\psi}, \tilde{g})+\Phi
$$

or

$$
\sigma(\psi, g)-\sigma(\tilde{\psi}, \tilde{g}) \leq \Phi
$$

Similarly, interchanging the roles of the couples $(\psi, g)$ and $(\tilde{\psi}, \tilde{g})$, we obtain

$$
\sigma(\tilde{\psi}, \tilde{g})-\sigma(\psi, g) \leq \Phi
$$

The proof for the discrete case is similar.

Remark 2.3. If $\psi=\widetilde{\psi}$, then (2.17) becomes

$$
\|u-\tilde{u}\|_{L^{\infty}(\Omega)} \leq\|g-\tilde{g}\|_{L^{\infty}(\partial \Omega)} .
$$

Theorem 2.4 (cf. [5]). Under conditions (2.3) and (2.4), there exists a constant $C$ independent of $h$ such that

$$
\left\|u-u_{h}\right\|_{L^{\infty}(\Omega)} \leq C h^{2}|\ln h|^{2}
$$


2.2. The continuous Schwarz sequences. Consider the model obstacle problem: find $u \in K_{0}(g=0)$ such that

$$
a(u, v-u) \geq(f, v-u) \quad \forall v \in K_{0} .
$$

We decompose $\Omega$ into two overlapping polygonal subdomains $\Omega_{1}$ and $\Omega_{2}$ such that

$$
\Omega=\Omega_{1} \cup \Omega_{2}
$$

and $u$ satisfies the local regularity condition

$$
u / \Omega_{i} \in W^{2, p}\left(\Omega_{i}\right) ; \quad 2 \leq p<\infty .
$$

We denote by $\partial \Omega_{i}$ the boundary of $\Omega_{i}$, and $\Gamma_{i}=\partial \Omega_{i} \cap \Omega_{j}$. The intersection of $\bar{\Gamma}_{i}$ and $\bar{\Gamma}_{j}$; $i \neq j$ is assumed to be empty.

Choosing $u^{0}=\psi$, we respectively define the alternating Schwarz sequences $\left(u_{1}^{n+1}\right)$ on $\Omega_{1}$ such that $u_{1}^{n+1} \in K$ solves

$$
\begin{gathered}
a_{1}\left(u_{1}^{n+1}, v-u_{1}^{n+1}\right) \geq\left(f_{1}, v-u_{1}^{n}\right) \quad \forall v \in K, \\
u_{1}^{n+1}=u_{2}^{n} \quad \text { on } \Gamma_{1}, v=u_{2}^{n} \text { on } \Gamma_{1}
\end{gathered}
$$

and $\left(u_{2}^{n+1}\right)$ on $\Omega_{2}$ such that $u_{2}^{n+1} \in K$ solves

$$
\begin{gathered}
a_{2}\left(u_{2}^{n+1}, v-u_{2}^{n+1}\right) \geq\left(f_{2}, v-u_{2}^{n+1}\right) \quad \forall v \in K, \\
u_{2}^{n+1}=u_{1}^{n+1} \quad \text { on } \Gamma_{2} ; v=u_{1}^{n+1} \text { on } \Gamma_{2},
\end{gathered}
$$

where

$$
f_{i}=f \Omega_{\Omega_{i}}, \quad a_{i}(u, v)=\int_{\Omega_{i}}(\nabla u \nabla v) d x .
$$

The following geometrical convergence is due to Lions [9].

\subsection{Geometrical convergence.}

Theorem 2.5 (cf. [9]). The sequences $\left(u_{1}^{n+1}\right) ;\left(u_{2}^{n+1}\right) ; n \geq 0$ produced by the Schwarz alternating method converge geometrically to the solution $u$ of the obstacle problem (2.28). More precisely, there exist two constants $k_{1}, k_{2} \in(0,1)$ such that for all $n \geq 0$,

$$
\begin{aligned}
& \left\|u_{1}-u_{1}^{n+1}\right\|_{L^{\infty}\left(\Omega_{1}\right)} \leq k_{1}^{n} k_{2}^{n}\left\|u^{0}-u\right\|_{L^{\infty}\left(\Gamma_{1}\right)}, \\
& \left\|u_{2}-u_{2}^{n+1}\right\|_{L^{\infty}\left(\Omega_{2}\right)} \leq k_{1}^{n+1} k_{2}^{n}\left\|u^{0}-u\right\|_{L^{\infty}\left(\Gamma_{2}\right)},
\end{aligned}
$$

where $u_{i}=u / \Omega_{i}, i=1,2$.

2.4. The discretization. For $i=1,2$, let $\tau^{h_{i}}$ be a standard regular and quasi-uniform finite element triangulation in $\Omega_{i} ; h_{i}$, being the meshsize. We assume that the two triangulations are mutually independent on $\Omega_{1} \cap \Omega_{2}$ in the sense that a triangle belonging to one triangulation does not necessarily belong to the other. 
Let $V_{h_{i}}=V_{h_{i}}\left(\Omega_{i}\right)$ be the space of continuous piecewise linear functions on $\tau^{h_{i}}$ which vanish on $\partial \Omega \cap \partial \Omega_{i}$. For $w \in C\left(\bar{\Gamma}_{i}\right)$ we define

$$
V_{h_{i}}^{(w)}=\left\{v \in V_{h_{i}}: v=0 \text { on } \partial \Omega_{i} \cap \partial \Omega ; v=\pi_{h_{i}}(w) \text { on } \Gamma_{i}\right\}
$$

where $\pi_{h_{i}}$ denotes the interpolation operator on $\Gamma_{i}$.

We also assume that the respective matrices resulting from the discretizations of problems (2.31) and (2.32), are $M$-matrices. (see [4]).

We now define the discrete counterparts of the continuous Schwarz sequences defined in $(2.31)$ and (2.32), respectively by: $u_{1 h}^{n+1} \in V_{h_{1}}^{\left(u_{2 h}^{n}\right)}$ such that

$$
\begin{aligned}
a_{1}\left(u_{1 h}^{n+1}, v-u_{1 h}^{n+1}\right) & \geq\left(f_{1}, v-u_{1 h}^{n+1}\right) \quad \forall v \in V_{h_{1}}^{\left(u_{2 h}^{n}\right)} \\
u_{1 h}^{n+1} & \leq r_{h}, \quad v \leq r_{h} \psi
\end{aligned}
$$

and $u_{2 h}^{n+1} \in V_{h_{2}}^{\left(u_{1 h}^{n+1}\right)}$ such that

$$
\begin{aligned}
a_{2}\left(u_{2 h}^{n+1}, v-u_{2 h}^{n+1}\right) & \geq\left(f_{2}, v-u_{2 h}^{n+1}\right) \quad \forall v \in V_{h_{2}}^{\left(u_{1 h}^{n+1}\right)} \\
u_{2 h}^{n+1} & \leq r_{h}, \quad v \leq r_{h} \psi
\end{aligned}
$$

Remark 2.6. As the two meshes $\tau^{h_{1}}$ and $\tau^{h_{2}}$ are independent over the overlapping subdomains, it is impossible to formulate a global approximate problem which would be the direct discrete counterpart of problem (2.28).

\section{3. $L^{\infty}$-error analysis}

This section is devoted to the proof of the main result of the present paper. To that end we begin by introducing two discrete auxiliary sequences and prove a fundamental lemma.

3.1. Definition of two auxiliary sequences. For $\omega_{i h}^{0}=u_{i h}^{0}=r_{h} \psi ; i=1,2$, we define the sequences $\left(\omega_{1 h}^{n+1}\right)$ such that $\omega_{1 h}^{n+1} \in V_{h_{1}}^{\left(u_{2}^{n}\right)}$ solves

$$
\begin{gathered}
a_{1}\left(\omega_{1 h}^{n+1}, v-\omega_{1 h}^{n+1}\right) \geq\left(f_{1}, v-\omega_{1 h}^{n+1}\right) \quad \forall v \in V_{h_{1}}^{\left(u_{2}^{n}\right)} \\
\omega_{1 h}^{n+1} \leq r_{h} \psi, \quad v \leq r_{h} \psi
\end{gathered}
$$

and $\left(\omega_{2 h}^{n+1}\right)$ such that $\omega_{2 h}^{n+1} \in V_{h_{2}}^{\left(u_{1}^{n+1}\right)}$ solves

$$
\begin{gathered}
a_{1}\left(\omega_{2 h}^{n+1}, v-\omega_{2 h}^{n+1}\right) \geq\left(f_{2}, v-\omega_{2 h}^{n+1}\right) \quad \forall v \in V_{h_{2}}^{\left(u_{1}^{n+1}\right)}, \\
\omega_{2 h}^{n+1} \leq r_{h} \psi, \quad v \leq r_{h} \psi .
\end{gathered}
$$

Note that $\omega_{i h}^{n+1}$ is the finite element approximation of $u_{i}^{n+1}$ defined in (2.31), (2.32). 
Notation 1. From now on, we will adopt the following notations:

$$
\begin{array}{cl}
|\cdot|_{1}=\|\cdot\|_{L^{\infty}\left(\Gamma_{1}\right)}, & |\cdot|_{2}=\|\cdot\|_{L^{\infty}\left(\Gamma_{2}\right)}, \\
\|\cdot\|_{1}=\|\cdot\|_{L^{\infty}\left(\Omega_{1}\right)}, & \|\cdot\|_{2}=\|\cdot\|_{L^{\infty}\left(\Omega_{2}\right)}, \\
\pi_{h_{1}}=\pi_{h_{2}}=\pi_{h} .
\end{array}
$$

The following lemma will play a key role in proving the main result of this paper.

Lemma 3.1.

$$
\begin{aligned}
& \left\|u_{1}^{n+1}-u_{1 h}^{n+1}\right\|_{1} \leq \sum_{p=1}^{n+1}\left\|u_{1}^{p}-\omega_{1 h}^{p}\right\|_{1}+\sum_{p=0}^{n}\left\|u_{2}^{p}-\omega_{2 h}^{p}\right\|_{2}, \\
& \left\|u_{2}^{n+1}-u_{2 h}^{n+1}\right\|_{2} \leq \sum_{p=0}^{n+1}\left\|u_{2}^{p}-\omega_{2 h}^{p}\right\|_{2}+\sum_{p=1}^{n+1}\left\|u_{1}^{p}-\omega_{1 h}^{p}\right\|_{1} .
\end{aligned}
$$

Proof. The proof will be carried out by induction. In order to simplify the notations, we will take $h_{1}=h_{2}=h$.

Indeed, for $n=1$, using the discrete version of Remark 2.3, we get

$$
\begin{aligned}
\left\|u_{1}^{1}-u_{1 h}^{1}\right\|_{1} & \leq\left\|u_{1}^{1}-\omega_{1 h}^{1}\right\|_{1}+\left\|\omega_{1 h}^{1}-u_{1 h}^{1}\right\|_{1} \leq\left\|u_{1}^{1}-\omega_{1 h}^{1}\right\|_{1}+\left|\pi_{h} u_{2}^{0}-\pi_{h} u_{2 h}^{0}\right|_{1} \\
& \leq\left\|u_{1}^{1}-\omega_{1 h}^{1}\right\|_{1}+\left|u_{2}^{0}-u_{2 h}^{0}\right|_{1} \leq\left\|u_{1}^{1}-\omega_{1 h}^{1}\right\|_{1}+\left\|u_{2}^{0}-u_{2 h}^{0}\right\|_{2}, \\
\left\|u_{2}^{1}-u_{2 h}^{1}\right\|_{2} & \leq\left\|u_{2}^{1}-\omega_{2 h}^{1}\right\|_{2}+\left\|\omega_{2 h}^{1}-u_{2 h}^{1}\right\|_{2} \leq\left\|u_{2}^{1}-\omega_{2 h}^{1}\right\|_{2}+\left|\pi_{h} u_{1}^{1}-\pi_{h} u_{1 h}^{1}\right|_{2} \\
& \leq\left\|u_{2}^{1}-\omega_{2 h}^{1}\right\|_{2}+\left|u_{1}^{1}-u_{1 h}^{1}\right|_{2} \leq\left\|u_{2}^{1}-\omega_{2 h}^{1}\right\|_{2}+\left\|u_{1}^{1}-u_{1 h}^{1}\right\|_{1} \\
& \leq\left\|u_{2}^{1}-\omega_{2 h}^{1}\right\|_{2}+\left\|u_{1}^{1}-\omega_{1 h}^{1}\right\|_{1}+\left\|u_{2}^{0}-u_{2 h}^{0}\right\|_{2}
\end{aligned}
$$

so

$$
\begin{aligned}
& \left\|u_{1}^{1}-u_{1 h}^{1}\right\|_{1} \leq \sum_{p=1}^{1}\left\|u_{1}^{p}-\omega_{1 h}^{p}\right\|_{1}+\sum_{p=0}^{0}\left\|u_{2}^{p}-\omega_{2 h}^{p}\right\|_{2}, \\
& \left\|u_{2}^{1}-u_{2 h}^{1}\right\|_{2} \leq \sum_{p=0}^{1}\left\|u_{2}^{p}-\omega_{2 h}^{p}\right\|_{2}+\sum_{p=1}^{1}\left\|u_{1}^{p}-\omega_{1 h}^{p}\right\|_{1} .
\end{aligned}
$$

For $n=2$, using the discrete version of Remark 2.3, we have

$$
\begin{aligned}
\left\|u_{1}^{2}-u_{1 h}^{2}\right\|_{1} & \leq\left\|u_{1}^{2}-\omega_{1 h}^{2}\right\|_{1}+\left\|\omega_{1 h}^{2}-u_{1 h}^{2}\right\|_{1} \leq\left\|u_{1}^{2}-\omega_{1 h}^{2}\right\|_{1}+\left|\pi_{h} u_{2}^{1}-\pi_{h} u_{2 h}^{1}\right|_{1} \\
& \leq\left\|u_{1}^{2}-\omega_{1 h}^{2}\right\|_{1}+\left|u_{2}^{1}-u_{2 h}^{1}\right|_{1} \leq\left\|u_{1}^{2}-\omega_{1 h}^{2}\right\|_{1}+\left\|u_{2}^{1}-u_{2 h}^{1}\right\|_{2} \\
& \leq\left\|u_{1}^{2}-\omega_{1 h}^{2}\right\|_{1}+\left\|u_{2}^{1}-\omega_{2 h}^{1}\right\|_{2}+\left\|u_{1}^{1}-\omega_{1 h}^{1}\right\|_{1}+\left\|u_{2}^{0}-u_{2 h}^{0}\right\|_{2}, \\
\left\|u_{2}^{2}-u_{2 h}^{2}\right\|_{2} & \leq\left\|u_{2}^{2}-\omega_{2 h}^{2}\right\|_{2}+\left\|\omega_{2 h}^{2}-u_{2 h}^{2}\right\|_{2} \leq\left\|u_{2}^{2}-\omega_{2 h}^{2}\right\|_{2}+\left|\pi_{h} u_{1}^{2}-\pi_{h} u_{1 h}^{2}\right|_{1} \\
& \leq\left\|u_{2}^{2}-\omega_{2 h}^{2}\right\|_{2}+\left|u_{1}^{2}-u_{1 h}^{2}\right|_{1} \leq\left\|u_{2}^{2}-\omega_{2 h}^{2}\right\|_{2}+\left\|u_{1}^{2}-u_{1 h}^{2}\right\|_{2} \\
& \leq\left\|u_{2}^{2}-\omega_{2 h}^{2}\right\|_{2}+\left\|u_{1}^{2}-\omega_{1 h}^{2}\right\|_{1}+\left\|u_{2}^{1}-\omega_{2 h}^{1}\right\|_{2}+\left\|u_{1}^{1}-\omega_{1 h}^{1}\right\|_{1}+\left\|u_{2}^{0}-u_{2 h}^{0}\right\|_{2} .
\end{aligned}
$$


So

$$
\begin{aligned}
& \left\|u_{1}^{2}-u_{1 h}^{2}\right\|_{1} \leq \sum_{p=1}^{2}\left\|u_{1}^{p}-\omega_{1 h}^{p}\right\|_{1}+\sum_{p=0}^{1}\left\|u_{2}^{p}-\omega_{2 h}^{p}\right\|_{2} \\
& \left\|u_{2}^{2}-u_{2 h}^{2}\right\|_{1} \leq \sum_{p=0}^{2}\left\|u_{2}^{p}-\omega_{2 h}^{p}\right\|_{2}+\sum_{p=1}^{2}\left\|u_{1}^{p}-\omega_{1 h}^{p}\right\|_{1} .
\end{aligned}
$$

Let us now suppose that

$$
\left\|u_{2}^{n}-u_{2 h}^{n}\right\|_{2} \leq \sum_{p=0}^{n}\left\|u_{2}^{p}-\omega_{2 h}^{p}\right\|_{2}+\sum_{p=1}^{n}\left\|u_{1}^{p}-\omega_{1 h}^{p}\right\|_{1} .
$$

Then, using the discrete version of Remark 2.3 again, we get

$$
\begin{aligned}
\left\|u_{1}^{n+1}-u_{1 h}^{n+1}\right\|_{1} & \leq\left\|u_{1}^{n+1}-\omega_{1 h}^{n+1}\right\|_{1}+\left\|\omega_{1 h}^{n+1}-u_{1 h}^{n+1}\right\|_{1} \leq\left\|u_{1}^{n+1}-\omega_{1 h}^{n+1}\right\|_{1}+\left|\pi_{h} u_{2}^{n}-\pi_{h} u_{2 h}^{n}\right|_{1} \\
& \leq\left\|u_{1}^{n+1}-\omega_{1 h}^{n+1}\right\|_{1}+\left|u_{2}^{n}-u_{2 h}^{n}\right|_{1} \leq\left\|u_{1}^{n+1}-\omega_{1 h}^{n+1}\right\|_{1}+\left\|u_{2}^{n}-u_{2 h}^{n}\right\|_{2} \\
& \leq\left\|u_{1}^{n+1}-\omega_{1 h}^{n+1}\right\|_{1}+\sum_{p=0}^{n}\left\|u_{2}^{p}-\omega_{2 h}^{p}\right\|_{2}+\sum_{p=1}^{n}\left\|u_{1}^{p}-\omega_{1 h}^{p}\right\|_{1}
\end{aligned}
$$

and consequently,

$$
\left\|u_{1}^{n+1}-u_{1 h}^{n+1}\right\|_{1} \leq \sum_{p=1}^{n+1}\left\|u_{1}^{p}-\omega_{1 h}^{p}\right\|_{1}+\sum_{p=0}^{n}\left\|u_{2}^{p}-\omega_{2 h}^{p}\right\|_{2} .
$$

Likewise, using the above estimate, we get

$$
\begin{aligned}
\left\|u_{2}^{n+1}-u_{2 h}^{n+1}\right\|_{2} \leq & \left\|u_{2}^{n+1}-\omega_{2 h}^{n+1}\right\|_{2}+\left\|\omega_{2 h}^{n+1}-u_{2 h}^{n+1}\right\|_{2} \leq\left\|u_{2}^{n+1}-\omega_{2 h}^{n+1}\right\|_{2} \\
& +\left|\pi_{h} u_{1}^{n+1}-\pi_{h} u_{1 h}^{n+1}\right|_{2} \leq\left\|u_{2}^{n+1}-\omega_{2 h}^{n+1}\right\|_{2}+\left|u_{1}^{n+1}-u_{1 h}^{n+1}\right|_{2} \\
\leq & \left\|u_{2}^{n+1}-\omega_{2 h}^{n+1}\right\|_{2}+\left\|u_{1}^{n+1}-u_{1 h}^{n+1}\right\|_{1} \leq\left\|u_{2}^{n+1}-\omega_{2 h}^{n+1}\right\|_{2} \\
& +\sum_{p=1}^{n+1}\left\|u_{1}^{p}-\omega_{1 h}^{p}\right\|_{1}+\sum_{p=0}^{n}\left\|u_{2}^{p}-\omega_{2 h}^{p}\right\|_{2} .
\end{aligned}
$$

Hence,

$$
\left\|u_{2}^{n+1}-u_{2 h}^{n+1}\right\|_{2} \leq \sum_{p=0}^{n+1}\left\|u_{2}^{p}-\omega_{2 h}^{p}\right\|_{2}+\sum_{p=1}^{n+1}\left\|u_{1}^{p}-\omega_{1 h}^{p}\right\|_{1} .
$$

\section{2. $L^{\infty}$-error estimate.}

Theorem 3.2. Let $h=\max \left(h_{1}, h_{2}\right)$. Then, there exists a constant $C$ independent of both $h$ and $n$ such that

$$
\left\|u_{i}-u_{i h}^{n+1}\right\|_{L^{\infty}\left(\Omega_{i}\right)} \leq C h^{2}|\log h|^{3} ; \quad i=1,2 .
$$


Proof. Let us give the proof for $i=1$. The case $i=2$ is similar.

Indeed, let $\kappa=\max \left(k_{1}, k_{2}\right)$, then

$$
\begin{aligned}
\left\|u_{1}-u_{1 h}^{n+1}\right\|_{1} & \leq\left\|u_{1}-u_{1}^{n+1}\right\|_{1}+\left\|u_{1}^{n+1}-u_{1 h}^{n+1}\right\|_{1} \\
& \leq \kappa^{2 n}\left|u^{0}-u\right|_{1}+\left\|u_{1}^{n+1}-u_{1 h}^{n+1}\right\|_{1} \\
& \leq \kappa^{2 n}\left|u^{0}-u\right|_{1}+\sum_{p=1}^{n+1}\left\|u_{1}^{p}-\omega_{1 h}^{p}\right\|_{1}+\sum_{p=0}^{n}\left\|u_{2}^{p}-\omega_{2 h}^{p}\right\|_{2} \\
& \leq \kappa^{2 n}\left|u^{0}-u\right|_{1}+2(n+1) C h^{2}|\log h|^{2}
\end{aligned}
$$

where we have used Theorem 2.5, Lemma 3.1, and Theorem 2.4, respectively.

Now setting

$$
\kappa^{2 n} \leq h^{2}
$$

we obtain

$$
\left\|u_{1}-u_{1 h}^{n+1}\right\|_{1} \leq C h^{2}|\log h|^{3}
$$

which is the desired error estimate.

3.3. The equation case. The analysis developed above remains valid for the equation problem $(\psi=\infty)$. Consequently, the error estimate (3.14) becomes

$$
\left\|u_{i}-u_{i h}^{n+1}\right\|_{L^{\infty}\left(\Omega_{i}\right)} \leq C h^{2}|\log h|^{2} ; \quad i=1,2 .
$$

Remark 3.3. The reduction constant $k$ can be quite close to one if the overlapping region is thin. Therefore, to ensure a good accuracy of the approximation, this region must be large enough.

\section{References}

[1] L. Badea, On the Schwarz alternating method with more than two subdomains for nonlinear monotone problems, SIAM Journal on Numerical Analysis 28 (1991), no. 1, 179-204.

[2] M. Boulbrachene, Ph. Cortey-Dumont, and J.-C. Miellou, Mixing finite elements and finite differences in a subdomain method, First International Symposium on Domain Decomposition Methods for Partial Differential Equations (Paris, 1987), SIAM, Philadelphia, 1988, pp. 198-216.

[3] X.-C. Cai, T. P. Mathew, and M. V. Sarkis, Maximum norm analysis of overlapping nonmatching grid discretizations of elliptic equations, SIAM Journal on Numerical Analysis 37 (2000), no. 5, $1709-1728$.

[4] P. G. Ciarlet and P.-A. Raviart, Maximum principle and uniform convergence for the finite element method, Computer Methods in Applied Mechanics and Engineering 2 (1973), no. 1, 17-31.

[5] Ph. Cortey-Dumont, On finite element approximation in the $L^{\infty}$-norm of variational inequalities, Numerische Mathematik 47 (1985), no. 1, 45-57.

[6] Yu. A. Kuznetsov, P. Neittaanmäki, and P. Tarvainen, Overlapping domain decomposition methods for the obstacle problem, Domain Decomposition Methods in Science and Engineering (Como, 1992) (A. Quarteroni, J. Périaux, Yu. A. Kuznetsov, and O. B. Widlund, eds.), Contemp. Math., vol. 157, American Mathematical Society, Rhode Island, 1994, pp. 271-277. 
[7] __ Schwarz methods for obstacle problems with convection-diffusion operators, Domain Decomposition Methods in Scientific and Engineering Computing (University Park, Pa, 1993) (D. E. Keyes and J. Xu, eds.), Contemp. Math., vol. 180, American Mathematical Society, Rhode Island, 1994, pp. 251-256.

[8] P.-L. Lions, On the Schwarz alternating method. I, First International Symposium on Domain Decomposition Methods for Partial Differential Equations (Paris, 1987), SIAM, Philadelphia, 1988, pp. 1-42.

[9] _ On the Schwarz alternating method. II. Stochastic interpretation and order properties, Domain Decomposition Methods (Los Angeles, Calif, 1988), SIAM, Philadelphia, 1989, pp. 4770.

[10] T. P. Mathew and G. Russo, Maximum norm stability of difference schemes for parabolic equations on overset nonmatching space-time grids, Mathematics of Computation 72 (2003), no. 242, 619656.

[11] J. Zeng and S. Zhou, On monotone and geometric convergence of Schwarz methods for two-sided obstacle problems, SIAM Journal on Numerical Analysis 35 (1998), no. 2, 600-616.

M. Boulbrachene: Department of Mathematics, College of Science, Sultan Qaboos University,

P.O. Box 36, Muscat 123, Oman

E-mail address: boulbrac@squ.edu.om

S. Saadi: Departement de Mathematiques, Faculte des Sciences, Universite Badji Mokhtar, BP 12 Annaba, Algerie

E-mail address: signor_2000@yahoo.fr 